\title{
Impact of temporal scales and recurrent mobility patterns on the unfolding of epidemics
}

\author{
David Soriano-Paños ${ }^{1,2}$, Gourab Ghoshal ${ }^{3,4}$, Alex Arenas ${ }^{5}$ \\ and Jesús Gómez-Gardeñes ${ }^{1,2}$ \\ ${ }^{1}$ Department of Condensed Matter Physics, University of Zaragoza, Zaragoza \\ 50010, Spain. \\ 2 GOTHAM lab, Institute for Biocomputation and Physics of Complex Systems, \\ University of Zaragoza, Zaragoza 50018, Spain. \\ 3 Department of Physics \& Astronomy, University of Rochester, Rochester, NY \\ 14627, USA. \\ ${ }^{4}$ Goergen Institute for Data Science, University of Rochester, Rochester, NY \\ 14627, USA. \\ ${ }^{5}$ Departament d'Enginyeria Informàtica i Matemàtiques, Universitat Rovira i \\ Virgili, Tarragona 43007, Spain.
}

September 2019

\begin{abstract}
Human mobility plays a key role on the transformation of local disease outbreaks into global pandemics. Indeed, the inclusion of population movement into epidemic models has become mandatory for understanding current epidemic episodes as well as designing efficient prevention policies. Following this challenge, here we develop a Markovian framework that enables to address the impact of recurrent mobility patterns on epidemic onset at different temporal scales. The formalism is validated by comparing its predictions with results from mechanistic simulations. The fair agreement between theory and numerical simulations, enables us to derive an analytical expression for the epidemic threshold, capturing the critical conditions triggering epidemic outbreaks. Finally, by performing an exhaustive analysis of this epidemic threshold, we reveal that the impact of tuning human mobility on the emergence of diseases is strongly affected by the temporal scales associated to both epidemiological and mobility processes.
\end{abstract}

\section{Introduction}

Despite the benefits associated with the development of human activity, globalization entails, as a counterpart, the acceleration of epidemic processes and its geographical expansion, turning local outbreaks into global epidemics [1]. Two main drivers of these changes in the global epidemic landscape are the substantially increased human mobility due to long-haul flights [2-5] and the effects of climate change [6-8]. As a direct consequence of these two factors, during the last decades, we have been witness to the first reported autochthonous case of Dengue in Europe $[9,10]$ and the rapid expansion of Chikungunya virus across America [11]. Thus, it is clear that the inclusion of human activity patterns, in particular those associated to mobility, in the formulation of epidemic models is mandatory for achieving accurate descriptions of actual epidemic scenarios. 
Spurred by the relevance of human mobility patterns, many efforts have been devoted to determine the role played by human displacements in shaping the onset of epidemics. On the one hand, sophisticated agent-based models [12-15] have been proposed to simulate real epidemic scenarios. These models have been very useful to unveil the most exposed areas to single epidemic outbreaks, thus enabling the design of policies to reduce their impact accordingly. Nevertheless, the extensive information required to address each particular case and the number of parameters involved in these models make it difficult to obtain a clear interpretation about the impact of human mobility and its generalization to address different epidemic scenarios. On the other hand, for the sake of obtaining more general insights, theoretical frameworks have been also proposed to address the effects of human mobility on epidemic spreading. In this sense, the most extended way of incorporating human mobility patterns into epidemic modeling [16-18] is the use of metapopulations [19-22]. Metapopulations, originally introduced in the field of ecology, are networks whose nodes are identified with patches or areas across which a set of agents moves according to the flows dictated by the network topology.

Theoretical works usually contain assumptions limiting their capability of fairly reproducing the spatiotemporal unfolding of epidemics. For this reason, epidemic models based on metapopulations have been continuously refined to bridge this gap between theory and agent-based simulations. Thus, early models considering agents as random walkers [23-26] have been improved to include more realistic situations such as the recurrent nature of human mobility [27-32] or the coexistence of different mobility patterns $[33,34]$ within a population. In particular, recently we proposed a framework called MIR (Movement-Interaction-Return) model [35] which allows the use of real data about the population distribution as well as their recurrent mobility patterns, enabling the assessment of the impact of daily movements on the spread of diseases across a given city. A limitation of this model, is that considers a single time scale associated with movement, precluding the ability to assess the impact of the trip-duration on epidemic spreading. The aim of this paper is to overcome this limitation by extending the original MIR model to incorporate different temporal scales affecting mobility and epidemiological processes. For this purpose, we propose a generalized formalism which introduces a new parameter governing the time spent by agents outside their residence when displaying recurrent mobility patterns.

The manuscript is organized as follows: In Section II, we describe the main features of the model and present the theoretical framework based on Markovian equations that we compare with those derived for the original MIR model. In Section III, we validate the former equations by comparing their theoretical predictions with results from mechanistic simulations. Once validated, we take advantage of them and we introduce in Section IV a theoretical estimation of the epidemic threshold. In Section V, we study how the recovery time and the typical staying time associated to movements change the dependence of the epidemic threshold on human mobility. Finally, in Section VI, we discuss the relevance of our findings and their implications for the design of prevention policies. 


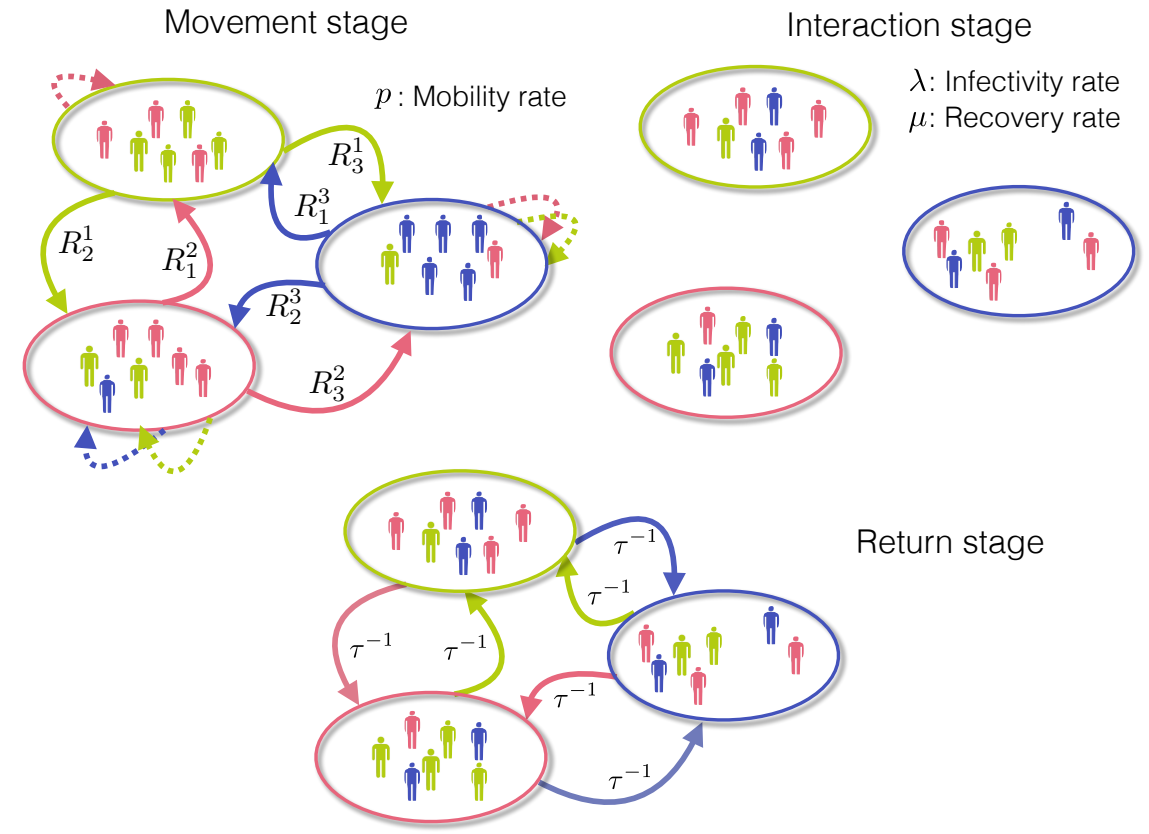

Figure 1. Schematic representation of the generalized MIR model. The agents color encodes their residence. Each time step involves three stages: $i$ ) Movement stage governed by the 3-dimensional tensor $\mathbf{M}$ computed according to Eq. (1). At this point, visitors outside their residence are forced to stay at their destination (dashed lines) whereas agents located at their residence are allowed to move according to the degree of mobility $p$ and the OD matrix $\mathbf{R}$. $i$ i) Interaction stage in which agents update their dynamical state according to the contagious rate $\lambda$ and the recovery one $\mu$. iii) Return stage where agents outside their residence can come back there with a probability $\tau^{-1}$.

\section{The model}

\subsection{Model assumptions}

We begin by describing the extended version of the MIR model. In Fig. 1 we show a schematic representation of the microscopic processes contained in our model. As the figure shows, a time step consists of three distinct stages. In the first stage, agents navigate the network with probability $p$; we note that unlike the original MIR model, agents are allowed to start outside their residence. Consequently, each patch at each time step, is (potentially) composed of individuals from different geographical areas. Given that residents and visitors usually display very different mobility patterns, one needs a more complex description of flows than the usual Origin-Destination (OD) matrices.

A general way to solve this problem is to introduce a 3-dimensional tensor $\mathbf{M}$ whose elements $M_{j k}^{i}$ describe the probability of an agent resident in $i$ moving from patch $j$ to patch $k$ [36]. Note that this general tensor allows us to recover several cases studied in the literature regarding human mobility. For instance, random walkers can be included by setting $M_{j k}^{i}=R_{j k}$ or back-and-forth movements are reflected by choosing $M_{j k}^{i}=\delta_{j}^{i} R_{j k}$ where $\mathbf{R}$ is the standard OD matrix. For the purposes of our 
study where we focus on the impact of duration of recurrent trips, $\mathbf{M}$ can be simplified and decomposed into:

$$
M_{j k}^{i}=\delta_{j}^{i} R_{k}^{j}+\left(1-\delta_{j}^{i}\right) \delta_{k}^{j} .
$$

The first term of Eq. (1) captures that movements from agents located in $i$ are governed by commuting flows encoded in the OD matrix $\mathbf{R}$, while the second term allows us to introduce the non-Markovian nature of permanence times in our Markovian equations via the introduction of self-loops governing the mobility of agents outside their residence. Once all the movements have taken place, agents start to interact. For the sake of simplicity, we deal with Susceptible-Infected-Susceptible (SIS) diseases and assume the population to be well-mixed so that agents located in the same patch all interact with each other. We define $\lambda$ as the probability that an infected agent transmits the pathogen to a susceptible one and $\mu$ as the probability that infected agents overcome the disease and become susceptible again.

Finally, to reflect the mostly commuting nature of human movement, visitors to a patch different from their residence either return back home or choose to stay in the patch with probability $\tau^{-1}$, i.e., $\tau$ encodes the typical time spent by visitors at their destination. Note that $\tau^{-1}=1$ recovers the original MIR model with back-and-forth daily movements whereas, for $\tau^{-1}=0$, agents loose information of their residence and are steadily redistributed across the neighboring areas.

\subsection{Model equations}

To build our formalism, we assume that the underlying metapopulation is divided into $P$ patches, each one with $N^{i}$ residents. The introduction of different temporal scales affecting mobility and the epidemiological processes requires us to account for the temporal evolution of populations crowding each patch. In this sense, we denote as $n_{j}^{i}(t)$ the number of residents in $i$ who are visiting $j$ at time $t$. Following the aforementioned microscopical rules, the evolution of these quantities reads:

$$
\begin{aligned}
n_{j}^{i}(t+1) & =\left(1-\delta_{j}^{i}\right)\left[\left(1-\tau^{-1}\right)\left(p R_{j}^{i} n_{i}^{i}(t)+n_{j}^{i}(t)\right)\right] \\
& +\delta_{j}^{i}\left[\left(1-p\left(1-R_{i}^{i}\right)\right) n_{i}^{i}(t)+\tau^{-1} \sum_{k \neq i}\left(p R_{k}^{i} n_{i}^{i}(t)+n_{k}^{i}(t)\right)(2)\right.
\end{aligned}
$$

The first line of the expression describes the evolution of the distribution of agents outside their residence. This is a sum of those that were already outside at time $t$ and decided not to return, and those moving from $i$ to $j$. The first term in the second line encodes those agents who decide not to leave their residence during the movement stage whereas the second one describes agents who start in a different place than their residence but come back home. As long as the relaxation time for the spread of diseases is much longer than the mobility time scale, one can assume that the distribution of agents across the metapopulation is given by the stationary solution of Eq. (2), thus considerably reducing the number of equations needed to track the evolution of diseases. Under this assumption, the number of agents residing in $i$, located in $j$ are given by:

$$
n_{j}^{i}=\left(1-\delta_{j}^{i}\right) \frac{N^{i} R_{j}^{i}}{1-R_{i}^{i}+\frac{\tau^{-1}}{p\left(1-\tau^{-1}\right)}}+\delta_{j}^{i} \frac{N^{i}}{1+\frac{p\left(1-R_{i}^{i}\right)\left(1-\tau^{-1}\right)}{\tau^{-1}}} .
$$

To track the evolution of the infection, we need to account for the number of infected residents from $i$ visiting $j$ at each time step, denoted as $\rho_{j}^{i}(t)$. Our formalism 
is thus composed of a set of $P \times E$ Markovian equations, where $E$ is the total number of connections of the underlying metapopulation. According to the assumptions of the model, the temporal evolution of $\rho_{j}^{i}(t)$ can be expressed as:

$$
\begin{aligned}
\rho_{j}^{i}(t+1) & =\delta_{j}^{i}[1-\mu]\left[\left(1-p\left(1-R_{i}^{i}\right)\right) \rho_{i}^{i}(t)+\tau^{-1} \sum_{l \neq j}\left(p R_{l}^{i} \rho_{i}^{i}(t)+\rho_{l}^{i}(t)\right)\right] \\
& +\delta_{j}^{i}\left(1-p\left(1-R_{i}^{i}\right)\right)\left(n_{i}^{i}-\rho_{i}^{i}(t)\right) \Pi_{i}(t) \\
& +\delta_{j}^{i} \tau^{-1} \sum_{l \neq j} \Pi_{l}(t)\left(p R_{l}^{i}\left(n_{i}^{i}-\rho_{i}^{i}(t)\right)+\left(n_{l}^{i}-\rho_{l}^{i}(t)\right)\right) \\
& +\left(1-\delta_{j}^{i}\right)(1-\mu)\left(1-\tau^{-1}\right)\left(p R_{j}^{i} \rho_{i}^{i}(t)+\rho_{j}^{i}(t)\right) \\
& +\left(1-\delta_{j}^{i}\right)\left(1-\tau^{-1}\right) \Pi_{j}(t)\left(p R_{j}^{i}\left(n_{i}^{i}-\rho_{i}^{i}(t)\right)+\left(n_{j}^{i}-\rho_{j}^{i}(t)\right)\right) .
\end{aligned}
$$

The first three lines describe the change in infected agents located at their residence; the first term includes all infected agents resident in $i$ and staying at their destination while not recovering from the infection, whereas the remaining terms describe contagions affecting susceptible individuals. Susceptible agents from $i$ can be infected inside their residence with probability $\Pi_{i}(t)$ or inside any other neighboring area $j$ with probability $\Pi_{j}$ before returning to $i$. Finally, the fourth and fifth lines encode the evolution of infected agents located outside their residence. Analogous to the former case, they include infected agents that do not recover and return(fourth line), and those who get infected at their destination with probability $\Pi_{j}(t)$ (fifth line). Following the well-mixed assumption for the interaction inside each patch, the probability of becoming infected inside a node $i$ at time $t, \Pi_{i}(t)$, reads:

$$
\Pi_{i}(t)=1-\prod_{j=1}^{N}\left(1-\lambda \frac{\left(\rho^{i n t}\right)_{i}^{j}(t)}{\left(n^{i n t}\right)_{i}^{j}}\right)^{\left(n^{i n t}\right)_{i}^{j}},
$$

where $\left(\rho^{i n t}\right)_{i}^{j}(t)$ denotes the number of infected individuals with residence in $j$ and interacting at $i$ at time $t$. Similarly, $\left(n^{i n t}\right)_{i}^{j}$ represents the total population from $j$ located at $i$ at the interaction stage. According to the mobility flows dictated by $\mathbf{M}$, both quantities are given by:

$$
\begin{aligned}
& \left(\rho^{i n t}\right)_{i}^{j}(t)=\delta_{j}^{i}\left(1-p\left(1-R_{j}^{j}\right)\right) \rho_{j}^{j}(t)+\left(1-\delta_{j}^{i}\right)\left(\rho_{i}^{j}+p R_{i}^{j} \rho_{j}^{j}(t)\right) \\
& \left(n^{i n t}\right)_{i}^{j}=\delta_{j}^{i}\left(1-p\left(1-R_{j}^{j}\right)\right) n_{j}^{j}+\left(1-\delta_{j}^{i}\right)\left(n_{i}^{j}+p R_{i}^{j} n_{j}^{j}\right) .
\end{aligned}
$$

\subsection{Limiting cases of the Markovian equations}

Let us analyze the two limiting cases of the former equations given by $\tau^{-1}=1$ and $\tau^{-1}=0$. In the latter case, the information of residence is no longer relevant and all agents are steadily redistributed across the metapopulation. In particular, the metapopulation turns into an effectively disconnected set of patches with a steady state population $N^{s t}$ given by

$$
N_{i}^{s t}=\sum_{j=1}^{P} N^{j} R_{i}^{j} .
$$

In the limit $\tau^{-1}=1$, we recover the original MIR model where Eq. (3) reduces to

$$
n_{j}^{i}=\delta_{j}^{i} N^{i}
$$



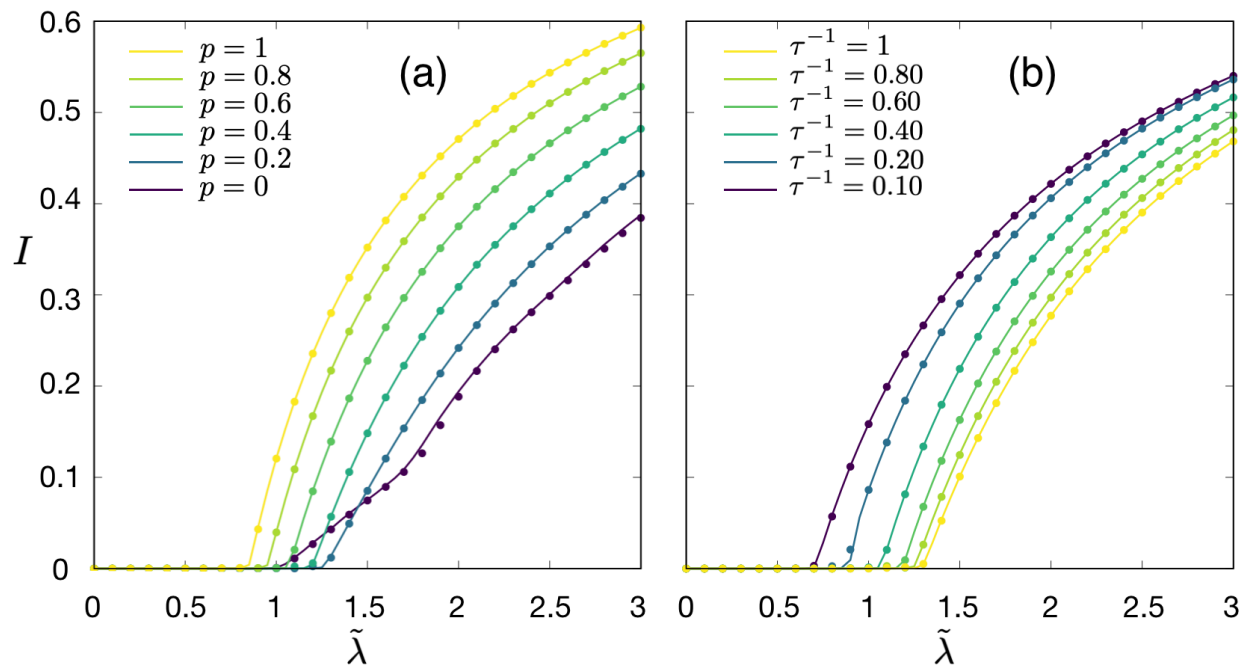

Figure 2. Panel a): Epidemic size (see text for details) $I$ as a function of the infectivity $\tilde{\lambda}$ and the mobility rate $p$ (color code). The return rate has been set to $\tau^{-1}=0.5$. Panel b) Epidemic size (see text for details) $I$ as a function of the infectivity $\tilde{\lambda}$ and the return rate $\tau^{-1}$ (color code). The mobility rate has been set to $p=0.5$. In both panels, solid lines represent theoretical predictions obtained by iterating the Eqs. (4-7) whereas dots show results from Monte Carlo simulations averaged over 100 realizations. The recovery rate in both panels has been set to $\mu=0.2$. Note that the infectivity $\tilde{\lambda}$ has been re-scaled by the epidemic threshold for the static case, i.e, $\tilde{\lambda}=\lambda / \lambda_{c}(p=0)=\lambda N_{\max } / \mu$.

indicating that all agents start from their residence. Thus, the Markovian model is reduced to $P$ equations, each one tracking the evolution of the fraction of infected agents associated to each patch, denoted as $\rho^{i}$. In particular, after normalizing by the population of each node, Eq. (4) turns into:

$$
\rho^{i}(t+1)=(1-\mu) \rho^{i}(t)+\left(1-\rho^{i}(t)\right)\left((1-p) \Pi_{i}(t)+p \sum_{j=1}^{N} R_{j}^{i} \Pi_{j}\right),(10
$$

thus reproducing the MIR model introduced in [35]. Increasing human mobility $p$ from $p=0$ always leads to an increase of the epidemic threshold $\lambda_{c}$, pointing out a non-trivial detrimental effect of mobility over the spread of diseases. In what follows, we validate the generalized Markovian equations and unveil the role that the newly introduced time scale $\tau$ plays on the aforementioned epidemic detriment driven by mobility. 


\section{Validation}

To validate Eqs.(4-7), we generate a Barabási-Albert synthetic metapopulation with $P=50$ patches and $\langle k\rangle=4$. Regarding agents' distribution, we assume that the population of each patch $i$ is assigned according to a uniform distribution spanning the range $N_{i} \in[300,1700]$. We first study the evolution of the epidemic size as a function of both mobility and epidemiological parameters. Here we define epidemic size as the fraction of the whole population that remains infected once the epidemic has reached the stationary state. Theoretically, we compute this indicator by introducing an infectious seed, encoded in the formalism by a non-zero initial configuration $\left\{\rho_{j}^{i}(0)\right\}$, and iterating Eqs. (4-7) until no fluctuations in the epidemic size are observed. On the other hand, to carry out the mechanistic simulations, we introduce a small number of infected individuals and let the population evolve according to the microscopic rules defined in Sec. II. Due to the stochastic nature of mechanistic simulations, all results are averaged over 100 realizations.

Fig. 2 shows the great agreement between both theoretical (curves) and numerical (dots) results. In addition, we observe that the epidemic threshold exhibits a nontrivial dependence on both parameters $p$ and $\tau^{-1}$. In particular, Fig. 2a reveals that, keeping fixed the permanence time $\tau(\tau=2$ in the figure), the variation of the mobility rate $p$ leads to the emergence of a non-monotonous behavior of the epidemic threshold, as illustrated in [35] for the original MIR model $(\tau=1)$. Namely, for low $p$ values, the epidemic threshold increases with the mobility, indicating that mobility is detrimental to the spread of diseases. However, when $p$ is high, the dependence is inverted and the increase of human mobility leads to a decrease of the epidemic threshold, thus boosting disease spreading. On the other hand, to analyze the influence of the temporal scale associated to movements, we set $p=0.5$ and study the effect of varying the return rate $\tau^{-1}$ in Fig. $2 \mathrm{~b}$. There, it becomes clear that by increasing the time associated to displacements fosters epidemic spreading since the epidemic threshold always decreases as $\tau$ increases.

\section{Analytical estimation of the epidemic threshold}

Here we aim at deriving an analytical expression of the epidemic threshold to capture the relevance of the different time scales for the onset of diseases. The epidemic threshold is defined as the minimum infectivity, that leads to population-wide epidemic outbreaks. To estimate its value, we make use of the next generation matrix method (NGM) [37], which leads to a compact expression for the basic reproduction number $R_{0}$.

First, to apply the NGM technique we turn the discrete equations into continuous ones. Since we are interested in the epidemic threshold, we assume that $\left(\rho^{i n t}\right)_{i}^{j} /\left(n^{i n t}\right)_{i}^{j}=\epsilon_{i}^{j} \ll 1 \forall(i, j)$. This allows us to linearize Eqs. (4-7) by neglecting terms of $\mathcal{O}\left(\epsilon^{2}\right)$. For the sake of readability, let us now explicitly express the evolution of infected agents inside and outside their residence. After linearizing the equations, the temporal dependence of the number of infected agents inside their residence $\dot{\rho}_{i}^{i}$ reads:

$$
\dot{\rho}_{i}^{i}=\underbrace{-\mu \rho_{i}^{i}-p\left(1-R_{i}^{i}\right)(1-\mu)\left(1-\tau^{-1}\right) \rho_{i}^{i}+\sum_{l \neq i}(1-\mu) \tau^{-1} \rho_{l}^{i}}_{V}
$$




$$
\begin{aligned}
& +\lambda \underbrace{\sum_{l}\left(1-p\left(1-R_{i}^{i}\right)\right) n_{i}^{i}\left[\delta_{i}^{l}\left(1-p\left(1-R_{i}^{i}\right) \rho_{i}^{i}+\left(1-\delta_{i}^{l}\right)\left(p R_{i}^{l} \rho_{l}^{l}+\rho_{i}^{l}\right)\right]\right.}_{F} \\
& +\lambda \underbrace{\tau^{-1} \sum_{m} \sum_{l \neq i}\left(p R_{l}^{i} n_{i}^{i}+n_{l}^{i}\right)\left[\delta_{m}^{l}\left[\left(1-p\left(1-R_{l}^{l}\right)\right) \rho_{l}^{l}\right]+\left(1-\delta_{m}^{l}\right)\left[p R_{l}^{m} \rho_{m}^{m}+\rho_{l}^{m}\right]\right]}_{F} .
\end{aligned}
$$

For $i \neq j$, the temporal evolution is given by:

$$
\begin{aligned}
\dot{\rho}_{j}^{i} & =\underbrace{-\mu \rho_{j}^{i}-\tau^{-1}(1-\mu) \rho_{j}^{i}+(1-\mu)\left(1-\tau^{-1}\right) p R_{j}^{i} \rho_{i}^{i}}_{V} \\
& +\underbrace{\lambda\left(1-\tau^{-1}\right)\left(p R_{j}^{i} n_{i}^{i}+n_{j}^{i}\right) \sum_{l}\left(\delta_{l}^{j}\left[1-p\left(1-R_{l}^{l}\right)\right] \rho_{j}^{j}+\left(1-\delta_{l}^{j}\right)\left[p R_{j}^{l} \rho_{l}^{l}+\rho_{j}^{l}\right]\right)}_{F} .
\end{aligned}
$$

Expressions (11-12) can be rewritten in a more compact way by defining two 4dimensional tensors $\mathbf{F}$ and $\mathbf{V}$. This way, the evolution of the number of infected individuals with residence in $i$ located at $j$ can be generally expressed as:

$$
\dot{\rho}_{j}^{i}=\left(\lambda F_{j m}^{i l}-V_{j m}^{i l}\right) \rho_{l}^{m} .
$$

Note that tensor $\mathbf{F}$ contains all the processes giving rise to an increase of the epidemic size whereas tensor $\mathbf{V}$ encodes the rest of transitions affecting infected agents. From the latter, the basic reproductive number $R_{0}$ can be obtained as:

$$
R_{0}=\frac{1}{\lambda \Lambda_{\max }\left(\mathbf{F V}^{-\mathbf{1}}\right)},
$$

where $\Lambda_{\max }\left(\mathbf{F V}^{-\mathbf{1}}\right)$ is the spectral radius of $\mathbf{F V}^{-\mathbf{1}}$. The epidemic threshold corresponds to the value of $\lambda$ leading to $R_{0}=1$, and therefore $\lambda_{c}$ is given by,

$$
\lambda_{c}=\frac{1}{\Lambda_{\max }\left(\mathbf{F V}^{-\mathbf{1}}\right)} .
$$

To study the effects of varying degrees of movement on the epidemic threshold, we first define $\tilde{\lambda}$ as $\tilde{\lambda}\left(p, \tau^{-1}\right)=\lambda_{c}\left(p, \tau^{-1}\right) / \lambda_{c}\left(0, \tau^{-1}\right)$, where the denominator is the threshold corresponding to the static case. Next, we consider its dependence on both $p$ and $\tau^{-1}$. Fig. 3 reveals the great accuracy of Eq. (15) for capturing the boundary between the disease-free region and the epidemic state. In addition, our theoretical estimation is also able to reproduce the non-monotonous impact of human mobility on the epidemic threshold and the promotion of epidemic spreading while increasing the permanence times at the destination.

\section{Interplay between mobility and epidemiological time scales}

In the previous section, while we obtained a theoretical expression of the epidemic threshold $\tilde{\lambda}$, its computation involves solving an eigenvalue problem of a $P^{2} \times P^{2}$ matrix, which clearly limits getting any insights on the role that each parameter plays. Instead, we adopt a strategy of finding simple heuristic arguments, allowing us to better understand the physical roots of the observed behavior of the epidemic 

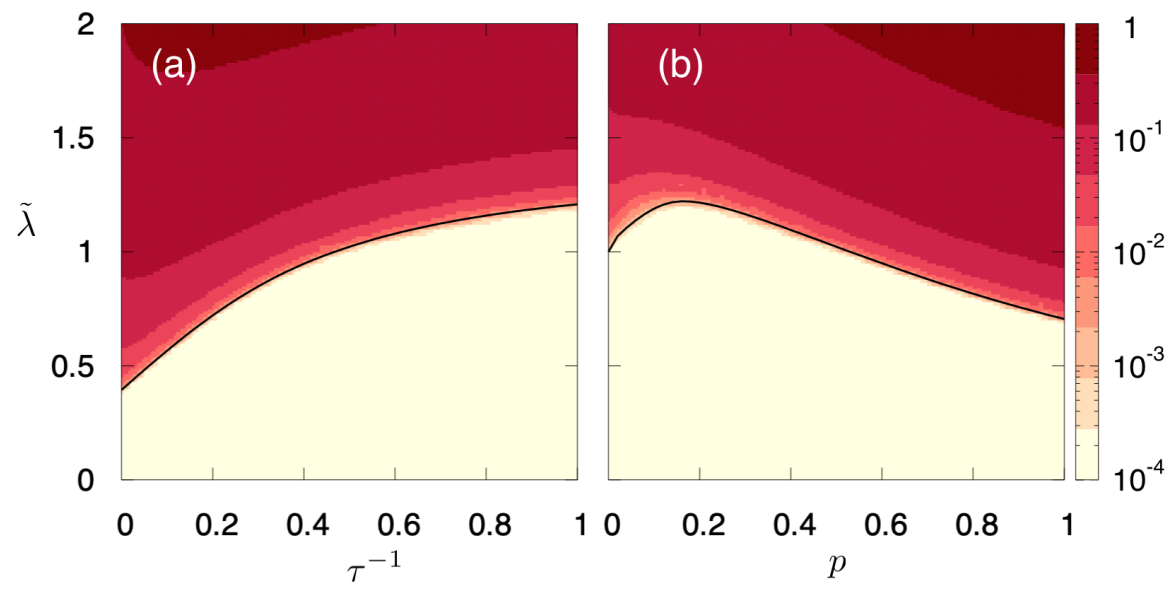

Figure 3. Left panel: Epidemic size (color code) as a function of the infectivity $\tilde{\lambda}$ and the probability of returning to home $\tau^{-1}$. The mobility rate $p$ has been set to $p=0.5$. Right panel: Epidemic size (color code) as a function of the infectivity $\tilde{\lambda}$ and the mobility rate $p$. The return rate has been set to $\tau^{-1}=0.50$. In both panels, the solid black line shows the estimation for the epidemic threshold $\lambda_{c}\left(p, \tau^{-1}\right)$ computed from Eq. (15). The recovery rate has been fixed to $\mu=0.2$.

threshold. Let us start by analyzing the static scenario in which nobody moves $(p=0)$ so all the agents only interact with the other residents in their associated patch. In this particular case, each patch displays a different epidemic threshold $\lambda_{c}^{i}$ given by

$$
\lambda_{c}^{i}=\frac{\mu}{N_{i}}
$$

and therefore the global epidemic threshold for the static case is,

$$
\lambda_{c}\left(p=0, \tau^{-1}\right)=\frac{\mu}{N_{\max }},
$$

where $N_{\max }$ is the number of residents in the most populated patch.

To unveil the role of the mobility $p$, we fix the return rate to $\tau^{-1}=0.5$ and represent in Fig. 4 the mobility network along with the population distribution for the static case for a non-zero value of the mobility. As the figure shows, mobility clearly promotes the accumulation of agents inside the most connected patches. Following our previous arguments, one could intuitively argue that the increase of mobility decreases the epidemic threshold due to the promotion of crowded areas that foster the spread of diseases. However, to understand the epidemic detriment observed in Fig. 3a, we should also consider that, at the individual level, increasing human mobility also facilitates the spatial diffusion of infected agents, thus preventing their accumulation inside those crowded subpopulations during their whole infectious period (of typical duration $\mu^{-1}$ time steps). Therefore, mobility promotes epidemic spreading because of the accumulation of agents in some areas but also hinders it by keeping infected agents away from vulnerable areas. As a consequence, the observed value of the epidemic threshold for each value of the mobility $p$ is the outcome of the competition between such opposite effects.

Interestingly, this trade-off is highly influenced by the return rate $\tau^{-1}$. In particular, the increase of the permanence time that visitors spend at their destination, 

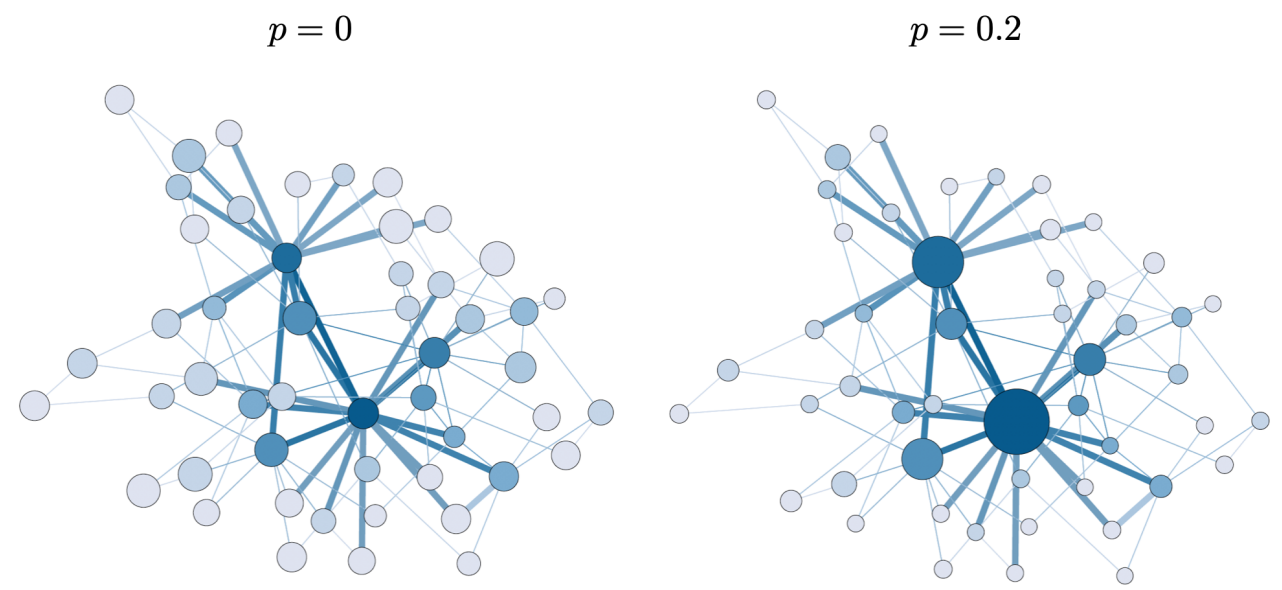

Figure 4. Barabási-Albert metapopulation governing human flows. The number of incoming connections of each patch is encoded in the color code ranging from the darker tones associated to highly connected patches to the brighter ones corresponding to barely connected areas. The size of the patches is proportional to the effective population interacting inside them. The mobility rate has been set to $p=0$ (Left) and $p=0.2$ (Right). The return rate is fixed to $\tau^{-1}=0.5$ in right panel. The number of residents in each area, say $i$, is assigned according to an uniform distribution within the range $N^{i} \in[300,1700]$.

i.e., decreasing $\tau^{-1}$, reduces the beneficial effect of the mobility, for it implies that infected agents have a higher probability of being in the most affected areas during their typical infectious time, $\mu^{-1}$, thus promoting a larger number of contagions. To confirm this phenomenon, we represent in Fig. 5a the dependence of the normalized epidemic threshold $\tilde{\lambda}_{c}$ on the mobility $p$ while tuning the permanence time $\tau^{-1}$. The figure makes it clear that for small time scales associated to human movements (high values of $\tau^{-1}$ ), the spatial diffusion of infected agents can compensate the accumulation of agents due to the mobility. Therefore, the overall result is a detriment of the epidemic spreading when increasing $p$. In contrast, for long permanence times, the dissemination of infected agents is clearly reduced and the creation of crowded sources of infection gains relevance, thus leading to the enhancement of epidemic spreading with mobility.

It is also worth studying the effects of the interplay between both epidemiological and mobility time scales in shaping epidemic outbreaks. To do so, we fix the return rate to $\tau^{-1}=0.1$ and plot in Fig. $5 \mathrm{~b} \tilde{\lambda}_{c}$ as a function of $p$ for several values of $\mu$. In this case, increasing the typical recovery time suppresses the effect of introducing larger time scales associated to human movements since epidemic detriment is observed only when $\mu<\tau^{-1}$. This is an expected result since, following our previous arguments, as long as the typical epidemiological time scale $\mu^{-1}$ is much longer than the one associated to movements, $\tau$, mobility allows an infected individual to visit different areas far from crowded subpopulations before overcoming the disease. In contrast, when $\mu^{-1}<\tau$, infected agents spend most of their epidemiological cycle inside one specific area. In this sense, the creation of contagions sources due to human mobility along with the continuous presence of infected agents lead to the decrease of the epidemic threshold when promoting human movement. 

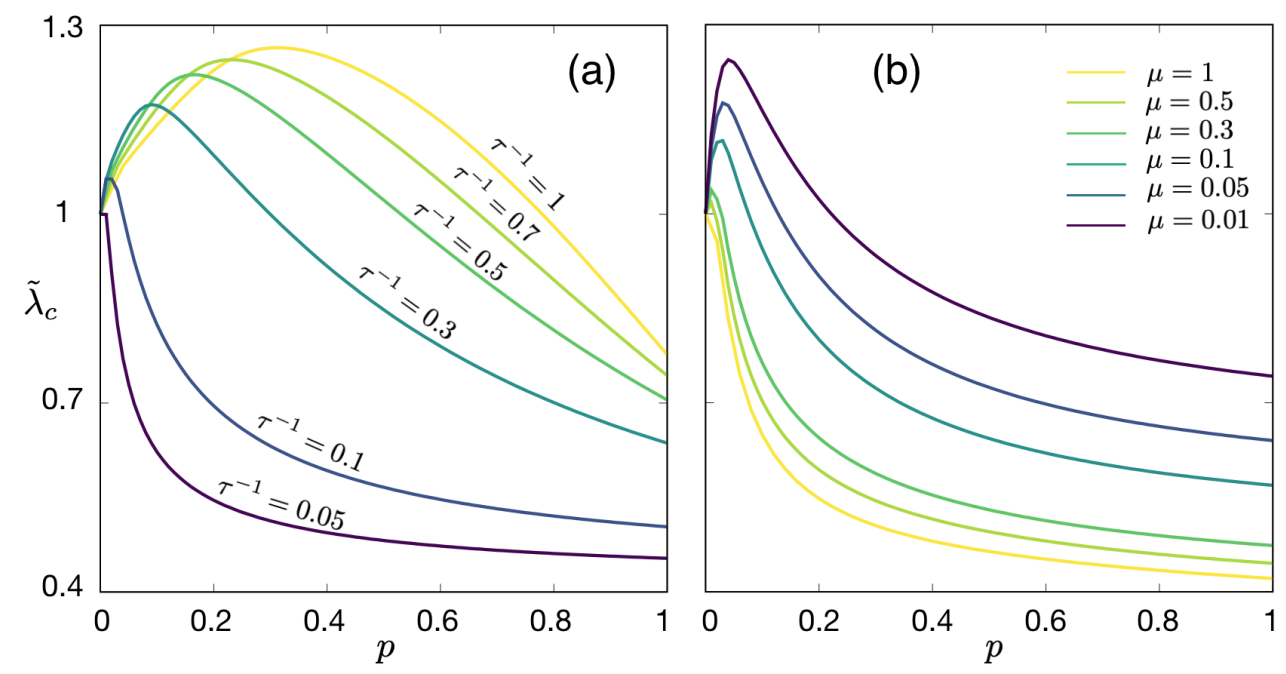

Figure 5. Left panel: Normalized epidemic threshold $\tilde{\lambda}_{c}$ as a function of the mobility for several values of the return rate $\tau^{-1}$ (color code). The recovery rate has been set to $\mu=0.2$. Right panel: $\tilde{\lambda}_{c}$ as a function of the mobility for several values of the recovery rate $\mu$. The return rate is fixed to $\tau^{-1}=0.10$.

Finally, we shed more light on the antagonistic character of the epidemic time scale $\mu^{-1}$ and the permanence time of human movements $\tau$. For this purpose, we study the surface $\tilde{\lambda}\left(\mu, \tau^{-1}\right)$ while setting $p=0.5$ in Fig. $6 \mathrm{a}$. We observe that the increase of the time scale governing recovery processes (decreasing $\mu$ ) leads to an increase in $\tilde{\lambda}_{c}$, thus diminishing the effect of prolonging the permanence times at the location of the contagion. This effect becomes more evident in Fig. $6 \mathrm{~b}$ where we explicitly show the dependence of $\tilde{\lambda}$ on $\tau^{-1}$. Increasing the permanence time (reducing $\tau^{-1}$ ) always boosts the spread of the disease by reducing the epidemic threshold. This effect is much more pronounced when the epidemic time scale is smaller than the typical trip duration.

\section{Conclusions}

In this manuscript we have studied the influence of recurrent human mobility patterns on the onset of epidemics at different temporal scales. The main contribution of this work is to provide a metapopulation framework to obtain theoretical insights about the role of different parameters related to human mobility, extending and complementing previous results [33,35]. Importantly, this framework allows to incorporate data about the population distribution (demography) and the back-and-forth mobility patterns from Origin-Destination matrices of real populations such as cities, regions and countries. The presented framework is formulated through a set of Markovian evolution equations that generalize those introduced in [35] by incorporating the time scale associated to the duration of trips, here represented by the permanence time in the destination nodes.

From a computational point of view, the Markovian framework introduced in this work constitutes a computational time-saving approach since the accuracy of the theoretical predictions, enables the monitoring of the spatiotemporal evolution of 

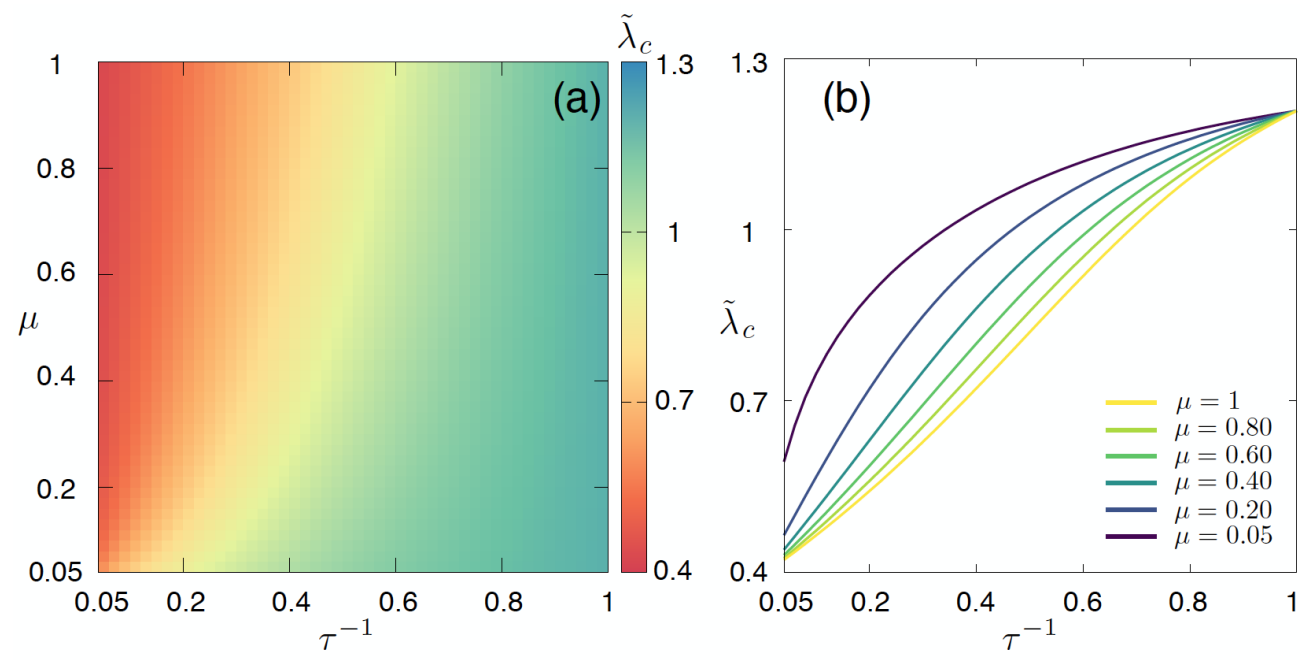

Figure 6. Left panel: Normalized epidemic threshold $\tilde{\lambda}_{c}$ (color code) as a function of the recovery rate $\mu$ and the return rate $\tau^{-1}$. Right panel: Dependence of the normalized epidemic threshold on the return rate $\tau^{-1}$ for several values of the recovery rate $\mu$. The mobility rate has been fixed in all panels to $p=0.5$.

SIS diseases, without the need of computationally costly agent-based simulations. Moreover, we have obtained a theoretical expression for the epidemic threshold, which allows us to determine the critical conditions triggering the onset of epidemics. Interestingly, our results indicate that the design of intervention policies to tackle epidemic outbreaks is strongly dependent on the interplay between different temporal scales associated to human movement and epidemic processes. The dependence of the epidemic threshold on mobility related parameters such as the frequency of trips or their duration, is strongly shaped by the typical recovery rate of the disease. Thus, the promotion of containment measures as it relates to mobility should be tailored to the specific nature of the disease to efficiently reduce their impact on society.

This work helps to bridge the gap between metapopulation models and real epidemic scenarios for which information about the demographical distribution of individuals and their mobility patterns is available. We note that some assumptions of the formalism are far from being realistic. For instance, agents associated with common geographical areas are indistinguishable and, in addition, movement patterns are assumed to be recurrent, neglecting more complex mobility patterns. Despite these assumptions, the presented mathematical formalism paves the way for the development of more realistic frameworks by considering other contagion mechanisms like those associated to vector-borne diseases [38-40], where the impact of human mobility is also shaped by other external factors such as vectors spatial distribution or climate change. Further refinements can be made by considering the multiplex nature of human flows [41-43], the existence of mobility patterns beyond recurrent movements [44-46] or the modification of human behaviors [47-49] as well as mobility patterns that respond to epidemic outbreaks $[16,50]$. 


\section{Acknowledgments}

D.S.-P. acknowledges financial support from Gobierno de Aragon through a doctoral fellowship. D.S.-P and J.G.G. acknowledge financial support from MINECO and FEDER funds through grants FIS2015-71582-C2 and FIS2017-87519-P, and Gobierno de Aragón with Fondo Social Europeo through grant E36-17R (FENOL). AA acknowledges support by MINECO and FEDER funds through grant FIS201571582-C2-1, Generalitat de Catalunya (grant 2017SGR896), Universitat Rovira i Virgili (grant 2017PFRURV-B2-41), ICREA Academia, and the James S. McDonnell Foundation (grant 220020325).

\section{References}

[1] J. F. Lindahl and D. Grace. The consequences of human actions on risks for infectious diseases: a review. Infect. Ecol. Epidemiol.Â 5, 30048 (2015).

[2] J. Brownstein, C.J. Wolfe and K.D. Mandl. Empirical evidence for the effect of airline travel on inter-regional influenza spread in the United State. PLoS Med 3, e40 (2006).

[3] A. Findlater and I. I. Bogoch. Human Mobility and the Global Spread of Infectious Diseases: A Focus on Air Travel. Trends in parasitology 34, 772 (2018).

[4] N.M Ferguson et al.. Strategies for mitigating an influenza pandemic. Nature 442, 448 (2006).

[5] R. Guimerá, S. Mossa, A. Turtschi, and L. A. N. Amaral The worldwide air transportation network: Anomalous centrality, community structure, and cities' global roles. Proc. Nat. Acad. Sci (USA) 102 7794-7799 (2005).

[6] E.P Hoberg and D.R Brooks. Evolution in action: climate change, biodiversity dynmaics and emerging infectious disease. Phil. Trans R Soc B 370, 20130553 (2015).

[7] K.D Lafferty. The ecology of climate change and infectious diseases. Ecology 90, 888 (2009).

[8] X. Wu et al. Impact of climate change on human infectious diseases: Empirical evidence and human adaptation. Environ. Int. ̂̂ Â 86, 14 (2016).

[9] Dengue outbreak in Madeira, Portugal. European Centre for Disease Prevention and Control (2013).

[10] Local transmission of dengue fever in France and Spain 2018. European Centre for Disease Prevention and Control (2018).

[11] H. Zeller, W. Van Bortel and B. Sundre. Chikungunya: Its History in Africa and Asia and Its Spread to New Regions in 2013-2014. Jour. Infect. Dis 214, 436 (2016)

[12] D. Balcan, B. Goncalves, H. Hu, J. J. Ramasco, V. Colizza and A.Vespignani. Modeling the spatial spread of infectious diseases: The GLobal Epidemic and Mobility computational model. Journal of Computational Science 1, 132-145 (2010).

[13] M. Tizzoni et al. Real-time numerical forecast of global epidemic spreading: case study of 2009 A/H1N1pdm. BMC Medicine 10165 (2012).

[14] Q. Zhang et al. Spread of Zika virus in the Americas. Proc. Nat. Acad. Sci (USA) 114, E4334 (2018).

[15] M. F. C. Gomes. Assessing the international spreading risk associated with the 2014 West African Ebola outbreak. PLoS Current Outbreaks (2014).

[16] C. Nicolaides, L. Cueto-Felgueroso and R. Juanes. The price of anarchy in mobility-driven contagion dynamics. Journal of The Royal Society Interface 10, 20130495 (2013).

[17] R. Pastor-Satorras, C. Castellano, P. Van Mieghem, and A. Vespignani. Epidemic processes in complex networks. Rev. Mod. Phys. 87, 925-979 (2015).

[18] W. Wang, M. Tang, H.E. Stanley and L.A. Braunstein. Unification of theoretical approaches for epidemic spreading on complex networks. Rep. Prog. Phys. 80, 036603 (2017).

[19] I. Hanski, and M.E. Gilpin. Metapopulation Biology: Ecology, Genetics, and Evolution. (Academic Press, 1997).

[20] I. Hanski, and O.E. Gaggiotti. Ecology, Genetics, and Evolution of Metapopulations.(Elsevier and Academic Press, 2004)

[21] M.J. Keeling, and P. Rohani. Modeling Infectious Diseases in Humans and Animals. (Princeton University Press, 2008).

[22] H.Ball el al.. Seven challenges for metapopulation models of epidemics, including households models. Epidemics 10, 63 (2015). 
[23] V. Colizza, R. Pastor-Satorras, and A. Vespignani. Reaction-diffusion processes and metapopulation models in heterogeneous networks. Nature Phys. 3, 276-282 (2007).

[24] V. Colizza, and A. Vespignani. Invasion threshold in heterogeneous metapopulation networks. Phys. Rev. Lett. 99, 148701 (2007).

[25] V. Colizza, and A. Vespignani. Epidemic modeling in metapopulation systems with heterogeneous coupling pattern: Theory and simulations. Journal of theoretical biology 251, 450-467 (2008).

[26] D. Balcan, V. Colizza, B. Gonçalves, H. Hao, J.J. Ramasco, and A. Vespignani. Multiscale mobility networks and the spatial spreading of infectious diseases- Proc. Nat. Acad. Sci (USA) 106, 21484-21489 (2009).

[27] D. Balcan, and A. Vespignani. Phase transitions in contagion processes mediated by recurrent mobility patterns. Nature Phys. 7, 581-586 (2011).

[28] V. Belik, T. Geisel, and D. Brockmann. Natural Human Mobility Patterns and Spatial Spread of Infectious Diseases. Phys. Rev. X 1, 1, 011001 (2011).

[29] D Balcan, and A Vespignani. Invasion threshold in structured populations with recurrent mobility patterns. J. Theor. Biol. 293, 87-100 (2011).

[30] V. Belik, T. Geisel, and D. Brockmann. Recurrent host mobility in spatial epidemics: beyond reaction-diffusion. Eur. Phys. J. B 84, 579-587 (2011).

[31] C. Poletto, M. Tizzoni, and V. Colizza. Human mobility and time spent at destination: impact on spatial epidemic spreading. J. Theor. Bio. 338, 41 (2013).

[32] C. Granell and P. Mucha. Epidemic spreading in localized environments with recurrent mobility patterns. Phys. Rev. E 97, 052302 (2018).

[33] D. Soriano-Paños, L. Lotero, A. Arenas, and J. Gómez-Gardeñes. Spreading processes in Multiplex Metapopulations Containing Different Mobility Networks. Phys. Rev. X 8, 031039 (2018).

[34] P. Bosetti et al.. Reducing measles risk in Turkey through social integration of Syrian refugees. arXiv:1901.04214 (2019).

[35] J. Gómez-Gardeñes, D. Soriano-Paños, and A. Arenas. Critical regimes driven by recurrent mobility patterns of reaction-diffusion processes in networks. Nature Phys. 14, 391-395 (2018).

[36] Note that, according to the our notation, superindexes denote the residence patch whereas subindexes account for the nodes where agents are currently placed and/or move to.

[37] O. Diekmann, J. A. P. Heesterbeek, and J. A. J. Metz. On the definition and computation of the basic reproduction ratio $\mathrm{R} 0$ in models for infectious diseases in heterogeneous populations. $J$. Math. Biol. 28, 365 (1990).

[38] A. Wesolowski et al. Quantifying the impact of human mobility on malaria. Science 338, 267-270 (2012).

[39] G. España et al. Exploring scenarios of Chikungunya mitigation with a data-driven agent-based model of the 2014-2016 outbreak in Colombia. Sci. Rep. 8, 12201 (2018).

[40] D.H. Barmak, C.O. Dorso, M. Otero and H.G. Solari. Dengue epidemics and human mobility. Phys. Rev. E 84, 011901 (2011).

[41] M. De Domenico, C. Granell, M. Porter, and A. Arenas. The physics of spreading processes in multilayer networks. Nat. Phys. 12, 901-906 (2016).

[42] L. Lotero, A. Cardillo, R. Hurtado and J. Gómez-Gardeñes. Several multiplexes in the same city: the role of socioeconomic differences in urban mobility, in Interconnected Networks (Springer, 2016).

[43] L. Lotero, R.G. Hurtado, L.M. Floría, and J. Gómez-Gardeñes. Rich do not rise early: Spatiotemporal patterns in the Mobility Networks of different Socio-economic classes. Royal Society Open Science 3, 150654 (2016).

[44] H. Barbosa-Filho, M. Barthelemy, G. Ghoshal, C.R. James, M. Lenormand, T. Louail, R. Menezes, J.J. Ramasco, F. Simini, and M. Tomasini. Human mobility: models and applications. Physics Reports 734, 1 (2017).

[45] J.T. Matamalas, M. De Domenico and A. Arenas. Assessing reliable human mobility patterns from higher order memory in mobile communications. J. R. Soc. Interface 13, 20160203 (2016).

[46] M. Lee, H. Barbosa, H. Youn, P. Holme, G. Ghoshal. Morphology of travel routes and the organization of cities. Nature Comm. 8, 2229 (2017).

[47] S. Funk, M. Salathé, and V.A. Jansen. Modelling the influence of human behaviour on the spread of infectious diseases: a review. Journal of the Royal Society Interface 7,1247 (2010).

[48] A. Cardillo, C. Reyes-Suárez, F. Naranjo, and J. Gómez-Gardeñes. Evolutionary vaccination dilemma in complex networks. Physical Review E 88, 032803 (2013).

[49] B. Steinegger, A. Cardillo, P. De Los Rios, J. Gómez-Gardeñes, and A. Arenas. Interplay between 
cost and benefits triggers nontrivial vaccination uptake. Physical Review E 97, 032308 (2018).

[50] S. Meloni et al.. Modeling human mobility responses to the large-scale spreading of infectious diseases. Sci. Rep. 9, 62 (2011). 